University of Nebraska - Lincoln

DigitalCommons@University of Nebraska - Lincoln

Nebraska Cooperative Fish \& Wildlife Research Nebraska Cooperative Fish \& Wildlife Research Unit -- Staff Publications

\title{
Variability between Scales: Predictors of Nomadism in Birds of an Australian Mediterraneanclimate Ecosystem
}

Craig R. Allen

U.S. Geological Survey, callen3@unl.edu

Denis A. Saunders

Clemson University

Follow this and additional works at: https://digitalcommons.unl.edu/ncfwrustaff

Part of the Other Environmental Sciences Commons

Allen, Craig R. and Saunders, Denis A., "Variability between Scales: Predictors of Nomadism in Birds of an Australian Mediterraneanclimate Ecosystem" (2002). Nebraska Cooperative Fish \& Wildlife Research Unit -- Staff Publications. 23.

https://digitalcommons.unl.edu/ncfwrustaff/23

This Article is brought to you for free and open access by the Nebraska Cooperative Fish \& Wildlife Research Unit at DigitalCommons@University of Nebraska - Lincoln. It has been accepted for inclusion in Nebraska Cooperative Fish \& Wildlife Research Unit -- Staff Publications by an authorized administrator of DigitalCommons@University of Nebraska - Lincoln. 


\title{
Variability between Scales: Predictors of Nomadism in Birds of an Australian Mediterranean- climate Ecosystem
}

\author{
Craig R. Allen ${ }^{1 *}$ and Denis A. Saunders ${ }^{2}$ \\ ${ }^{1}$ US Geological Survey, Biological Resources Division, South Carolina Cooperative Fish and Wildlife Research Unit, G27 Lehotsky, \\ Clemson University, Clemson, South Carolina 29634, USA; and ${ }^{2}$ CSIRO Sustainable Ecosystems, GPO Box 284, \\ Canberra ACT 2601, Australia
}

\begin{abstract}
Nomadism in animals is a response to resource distributions that are highly variable in time and space. Using the avian fauna of the Mediterranean-climate region of southcentral Australia, we tested a number of variables to determine if they predicted nomadism. These variables were species body mass, the distance in body mass terms to the edge of a body mass aggregation, and diet (for example, seeds, invertebrates, nectar, or plants). We utilized two different classifications of the avifauna that diverged in their definition of nomadic to build two different predictive models. Using both classifications, distance to the edge of a body mass aggregation was found to be a significant predictor of nomadism. There was also evidence that nomads tend to feed on nectar and tend to be large. The signifi-
\end{abstract}

\section{INTRODUCTION}

Nomadism is most prevalent in ecosystems with resources that are highly variable in space and time (Sinclair 1984). Nomadism is characterized by unpredictable movements in space and time that track unpredictable changes in resource distribution and abundance. Thus, nomadic species tend to occur disproportionately in arid or semi-arid ecosystems (Davies 1984). Nomadism in birds has been relatively little studied, and surprisingly little is known

Received 14 November 2000; accepted 27 June 2001

*Corresponding author; e-mail: Allencr@Clemson.edu cance of the variables body mass and diet (nectar) may reflect the greater energy requirements of large birds and the inherent variability of nectar as a food source. The significance of the variable distance to the edge of a body mass aggregation provides further evidence of inherent variability in resources between domains of scale. The edges of body mass aggregations are hypothesized to be areas of increased resource variability that reflect the transition from one scale of landscape pattern to another.

Key words: Australia; birds; body mass distributions; body mass pattern; landscape patterns; nomad; Mediterranean climate; scale; scale break; textural discontinuity hypothesis. of the ecological correlates of nomadism. Dean (1997) reported that nomadic species of the South African Karoo tended to be granivores, especially feeding on grass seeds, and further noted that nomadic species that feed on grass tended to be small. Davies (1984) found that nomads tend to feed on seeds or nectar, but also stated that some nomads are raptors, tracking rodent outbreaks. It is possible to imagine that nearly all resources in arid and semi-arid environments are highly variable in space and time and that their exploitation would lead to the evolution of nomadism, yet few species of birds are in fact nomadic, even where nomadism is relatively common. 
It is clear that nomadism is most common in ecosystems with high variation in resource abundance, often associated with low, variable, and unpredictable rainfall patterns (Davies 1984; Wiens 1991). For example, Keast (1968) documented a negative correlation between nomadism and rainfall in honeyeaters; Wiens (1991) suggested that the higher prevalence of nomadism in Australia as compared to North America was due to higher variability in rainfall; and nomadic South African larks have been reported to breed where rains have recently fallen (Maclean 1996). Nomadism in humans occurs in arid regions at the periphery of settled heartlands; the reversion of sedentary peoples to nomadic lifestyles may be a response to environmental changes leading to increased aridity (Rosen 1988). However, it is not known exactly what resources, and what other traits may be associated with and predictive of nomadism. Our purpose was to determine which-if any-of a number of variables suggested or theoretically predictive of nomadism, do so in the Mediterranean-climate region of southcentral Australia. Schodde (1981) reviewed the birds of the Mallee ecosystems of Australia, which includes the Lofty Block of the Interim Biogeographic Regionalization for Australia (Thackway and Cresswell 1995). He provided a list of species inhabiting this region and a categorization of species diet, and then classified species as nomadic or sedentary/migratory.

A number of variables may theoretically predispose a species toward nomadism. Specializing-in certain food sources that tend to be more ephemeral and unpredictable than others may lead to the evolution of a nomadic lifestyle. Thus, one set of variables (four diet types) focused upon the type of food resource utilized by a species. Body size correlates with a number of traits and ecological attributes of species (Eisenberg 1981), so we incorporated body size (mass) into our model. A final variable focused upon the link between the scalespecific nature of ecological pattern and structure and discontinuous body mass distributions (Holling 1992). The independent variables, and their basis for inclusion in our models, are further discussed below.

\section{METHODS}

Our area of interest was the Mediterranean-climate region of southcentral Australia, near the city of Adelaide, the capital of the state of South Australia, an area largely encompassed by the Lofty Block of the Interim Biogeographic Regionalization of Australia (Thackway and Cresswell 1995) (Figure 1).

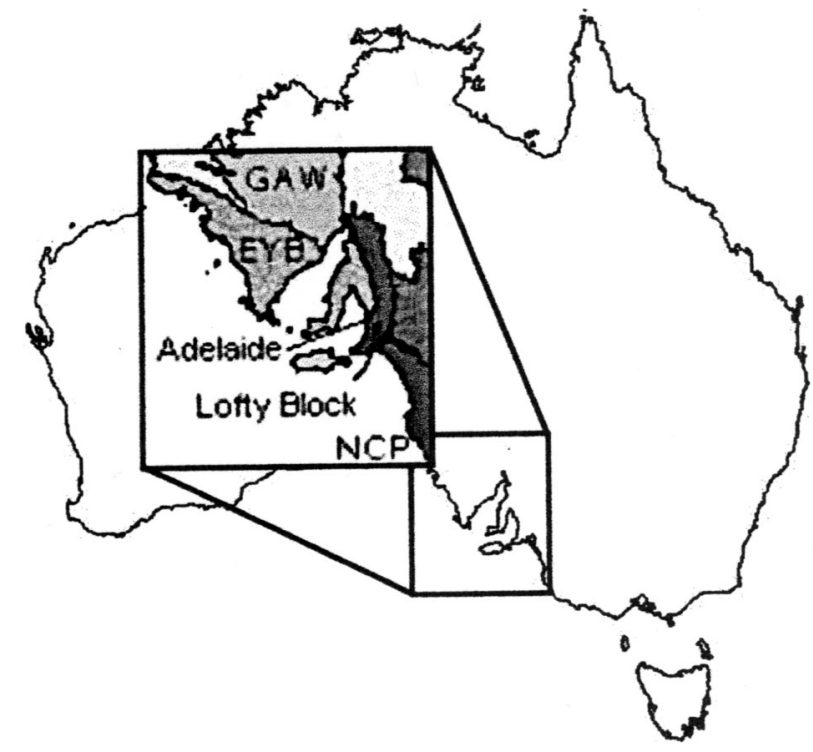

Figure 1. Australia and the Lofty Block region near the city of Adelaide in relation to surrounding biogeographic regions (from Thackway and Cresswell 1995). NCP, Naracoorte Coastal Plain; GAW, Gawler; EYB, Eyre and Yorke Block.

This region is characterized by cool, wet winters and hot, dry summers. It is an area of nearly $24,000 \mathrm{~km}^{2}$ that includes the southern Flinders Ranges, the Mount Lofty Ranges, and Kangaroo Island. The description of the Lofty Block given in the Interim Biogeographic Regionalization of Australia (Thackway and Cresswell 1995) is "eucalypt open forests and woodlands and heaths on mottled yellow and ironstone gravelly duplex soils in the wetter areas and red duplex soils in drier areas; now largely cleared for agriculture and urban development." The region has been extensively cleared of native vegetation, so that less than $5 \%$ of the former vegetation cover remains (Paton and others 2000).

We utilized published sources to determine our species list (Schodde 1981) and average body masses (Dunning 1993). Where published body mass estimates were not available, we used unpublished data provided by R. Schodde $(n=23)$. Species for which no body mass estimates were available were omitted from analyses $(n=6)$. We used two interpretations of nomadism. Our first interpretation utilized the published scheme of Schodde (1981), which provided a categorization of species as nomadic or sedentary. However, these classifications are general and may not always differentiate the situation where a species may be resident over part of its range but migrant, vagrant, or nomadic over other parts of its range. Therefore, we also 
used local expertise (that of D. Saunders and J. Reid, CSIRO Sustainable Ecosystems, Canberra, Australia) to categorize the birds of the Lofty Block and developed an independent list of nomadic species.

We used logistic regression (SAS Institute 1989) to determine significant predictors of nomadism, building two different models based upon the two different categorizations of nomadism. The binary response variable was whether or not a species was classified as nomadic. The independent variables were species body mass; the distance in body mass terms to the edge of a body mass aggregation (edge); and seed, invertebrate, nectar, or plant diet. We used backward selection logistic regression (Menard 1995), which provides an appropriate method for estimating a multiple regression when the dependent variable is binary (that is, 0 or 1 ). We set the significance level of independent variables to stay in the model at 0.1. We aggregated dietary categories of Schodde (1981) that were represented by too few species and where aggregation was biologically meaningful. Thus, the diet of Aquila audax was classified as invertebrate although it eats mammals, birds, and reptiles; the diets of Dicaeum hirundinaceum, Lichenostomus leucotis, Lichenostomus plumulus, and Cuculus pallidus were classified as nectar although they eat primarily fruit; and the diet of Glossopsitta porphyrocephala was classified as nectar although it feeds primarily on pollen.

The independent variables were chosen because there was a theoretical basis for believing that these variables may influence, or be related to, the occurrence of nomadism. The variable body mass was chosen because larger species have higher energy requirements (Lasiewski and Dawson 1967), which may require tracking of high-energy resources that appear unpredictably in Mediterranean-climate ecosystems.

The variable distance to a body mass aggregation edge (edge) was utilized because it has been suggested that the edge of body mass aggregations mirror scale breaks in ecological structure and are associated with high biological variability in attributes such as community composition. Allen and others (1999) documented a nonrandom distribution, in terms of the body mass pattern, of nonindigenous and declining species for three taxonomic replicates (herpetofauna, birds, and mammals) from the south Florida subecoregion and a similar pattern in nonindigenous species in birds and mammals from Mediterranean-climate Australia. Nomadism represents another type of biological variability theoretically associated with scale breaks.

To determine body mass structure-that is, the location of body mass aggregations ("lumps") (sensu Holling 1992) and gaps in the body mass distribution of the birds of our study area-we used computer simulations to locate significant discontinuities in the body mass distribution. Simulations compared actual body mass data with a null distribution established by estimating a continuous unimodal kernel distribution of the log-transformed data (Silverman 1981). Gaps were defined as areas between successive body masses that significantly exceeded the discontinuities generated by the continuous null distribution (Restrepo and others 1997). A species aggregation was a grouping of three or more species with body masses not exceeding the expectation of the null distribution. Each species aggregation, or lump, is defined by two endpoint species and their respective body masses. Thus, the variable distance to body mass aggregation edge is measured as the distance in body mass units to the nearest body mass aggregation defining (endpoint) species. It is assumed that species within an aggregation perceive and exploit their environment at the same ecological scale, which differs from species in other body mass aggregations (Peterson and others 1998; Allen and others 1999).

The variables seed, invertebrate, nectar, or plant related to the predominant diet of the species, as classified by Schodde (1981). Since different resources vary in their reliability and persistence over space and time, diet may be related to nomadism.

\section{RESULTS}

The avian species list for the Adelaide region Mallee of southcentral Australia, as published by Schodde, included 130 native species. However, we were unable to find body mass estimates for six species (Sericornus pyrrhopygius, Plectorhyncha lanceolata, Pardalotus xanthopygus, Emblema guttata, Struthidea cinerea, Strepera versicolor) and therefore eliminated them from our analyses. Schodde classified 76 species as nonnomadic and 48 species as nomadic (Table 1). Saunders and Reid provided a classification that differed from that of Schodde (Table 1) and classified only 24 species as nomadic. Differences between the Saunders and Reid classification and the Schodde classification were not restricted to reclassifying half of Schodde's nomadic species as "not nomadic" but also included classifying species considered resident by Schodde as nomadic. The diet of 60 species consisted primarily of seeds; the diet of 41 was classified as consisting primarily of invertebrates; 16 were classified as nectar feeders; and seven fed primarily on other plant material (Schodde 1981) (Table 1). 
Table 1. The Bird Community of the Mediterranean-Climate Mallee/Lofty Block near Adelaide, Southcentral Australia

\begin{tabular}{|c|c|c|c|c|c|c|}
\hline Species & $\begin{array}{l}\text { Nomad } \\
\text { Status } \\
\text { (Schodde) }\end{array}$ & $\begin{array}{l}\text { Nomad } \\
\text { Status (Reid) }\end{array}$ & Diet & $\begin{array}{l}\text { Log body } \\
\text { mass }\end{array}$ & Lump & Edge \\
\hline \multicolumn{7}{|l|}{ Smicrornis brevirostris } \\
\hline Weebill & 2 & 2 & seeds & 0.70757 & 1 & 0 \\
\hline \multicolumn{7}{|l|}{ Acanthiza nana } \\
\hline Yellow Thornbill & 2 & 2 & seeds & 0.77085 & 1 & 0 \\
\hline \multicolumn{7}{|l|}{ Acanthiza uropygialis } \\
\hline Thornbill & 2 & 2 & seeds & 0.87448 & 2 & 0 \\
\hline \multicolumn{7}{|l|}{ Acanthiza reguloides } \\
\hline Buff-rumped Thornbill & 1 & 2 & seeds & 0.87506 & 2 & 0.00579 \\
\hline \multicolumn{7}{|l|}{ Acanthiza apicalis } \\
\hline Inland Thornbill & 2 & 2 & seeds & 0.88081 & 2 & 0.06332 \\
\hline \multicolumn{7}{|l|}{ Certhionyx niger } \\
\hline Black Honeyeater & 1 & 1 & seeds & 0.89209 & 2 & 0.17612 \\
\hline \multicolumn{7}{|l|}{ Petroica goodenovii } \\
\hline Red-capped Robin & 2 & 2 & inverts & 0.90309 & 2 & 0.28608 \\
\hline \multicolumn{7}{|l|}{ Rhipidura fuliginosa } \\
\hline Grey Fantail & 2 & 2 & inverts & 0.90309 & 2 & 0.28608 \\
\hline \multicolumn{7}{|l|}{ Malurus lamberti } \\
\hline Variegated Fairy-wren & 2 & 2 & seeds & 0.90309 & 2 & 0.28608 \\
\hline \multicolumn{7}{|l|}{ Dicaeum hirundinaceum } \\
\hline Mistletoebird & 1 & 2 & nectar & 0.90309 & 2 & 0.28608 \\
\hline \multicolumn{7}{|l|}{ Acanthiza chrysorrhoa } \\
\hline \multicolumn{7}{|l|}{ Yellow-rumped } \\
\hline Thornbill & 2 & 2 & seeds & 0.94448 & 2 & 0.70001 \\
\hline \multicolumn{7}{|l|}{ Pardalotus punctatus } \\
\hline Spotted Pardalote & 1 & 1 & plant & 0.96379 & 2 & 0.89306 \\
\hline \multicolumn{7}{|l|}{ Petroica multicolor } \\
\hline Scarlet Robin & 2 & 2 & inverts & 0.98227 & 2 & 1.07789 \\
\hline \multicolumn{7}{|l|}{ Malurus splendens } \\
\hline Splendid Fairy-wren & 2 & 2 & seeds & 1 & 2 & 1.10590 \\
\hline \multicolumn{7}{|l|}{ Ephthianura tricolor } \\
\hline Crimson Chat & 2 & 1 & seeds & 1.02407 & 2 & 0.86515 \\
\hline \multicolumn{7}{|l|}{ Pyrrholaemus brunneus } \\
\hline Redthroat & 2 & 2 & seeds & 1.05231 & 2 & 0.58281 \\
\hline Hirundo ariel Fairy Martin & 2 & 2 & inverts & 1.05308 & 2 & 0.57511 \\
\hline \multicolumn{7}{|l|}{ Daphoenositta chrysoptera } \\
\hline Varied Sittella & 1 & 2 & inverts & 1.07555 & 2 & 0.35043 \\
\hline \multicolumn{7}{|l|}{ Pardalotus striatus } \\
\hline Striated Pardalote & 2 & 1 & inverts & 1.08636 & 2 & 0.24230 \\
\hline \multicolumn{7}{|l|}{ Aphelocephala leucopsis } \\
\hline Southern Whiteface & 2 & 2 & seeds & 1.10140 & 2 & 0.09186 \\
\hline Sericornis frontalis & & & & & & \\
\hline White-browed & & & & & & \\
\hline Scrubwren & 2 & 2 & seeds & 1.10721 & 2 & 0.03380 \\
\hline Taeniopygia guttata & & & & & & \\
\hline Zebra Finch & 1 & 1 & seeds & 1.10992 & 2 & 0.00674 \\
\hline Zosterops lateralis & & & & & & \\
\hline Silvereye & 1 & 1 & nectar & 1.11059 & 2 & 0 \\
\hline Hylacola cauta & & & & & & \\
\hline Shy Heathwren & 2 & 2 & seeds & 1.15290 & 3 & 0 \\
\hline
\end{tabular}


Table 1. (Continued)

\begin{tabular}{|c|c|c|c|c|c|c|}
\hline Species & $\begin{array}{l}\text { Nomad } \\
\text { Status } \\
\text { (Schodde) }\end{array}$ & $\begin{array}{l}\text { Nomad } \\
\text { Status (Reid) }\end{array}$ & Diet & $\begin{array}{l}\text { Log body } \\
\text { mass }\end{array}$ & Lump & Edge \\
\hline \multicolumn{7}{|l|}{ Melithreptus brevirostris } \\
\hline Honeyeater & 1 & 2 & nectar & 1.16435 & 3 & 0.11453 \\
\hline \multicolumn{7}{|l|}{ Hirundo neoxena } \\
\hline Welcome Swallow & 2 & 2 & inverts & 1.16731734 & 3 & 0.144177 \\
\hline \multicolumn{7}{|l|}{ Cheramoeca leucosternus } \\
\hline \multicolumn{7}{|l|}{ Hirundo nigricans } \\
\hline Jacky Winter & 2 & 2 & inverts & 1.19589965 & 3 & 0.16288 \\
\hline \multicolumn{7}{|l|}{ Amytornis striatus } \\
\hline $\begin{array}{l}\text { Striated Grasswren } \\
\text { Lichenostomus ornatus }\end{array}$ & 2 & 2 & inverts & 1.2121876 & 3 & 0 \\
\hline Yellow-plumed & & & & & & \\
\hline Honeyeater & 2 & 2 & nectar & 1.25042 & 4 & 0 \\
\hline \multicolumn{7}{|l|}{ Phylidonyris albifrons } \\
\hline Honeyeater & 1 & 1 & nectar & 1.25527251 & 4 & 0.048525 \\
\hline \multicolumn{7}{|l|}{ Pachycephala rufiventris } \\
\hline \multicolumn{6}{|l|}{ Lichenostomus plumulus } & 0.072586 \\
\hline $\begin{array}{l}\text { Grey-fronted } \\
\text { Honeyeater }\end{array}$ & 2 & 2 & nectar & 1.26007139 & 4 & 0.096514 \\
\hline \multicolumn{7}{|l|}{ Phylidonyris melanops } \\
\hline $\begin{array}{l}\text { Tawny-crowned } \\
\text { Honeyeater }\end{array}$ & 2 & 2 & nectar & 1.26717173 & 4 & 0.167517 \\
\hline \multicolumn{7}{|l|}{$\begin{array}{l}\text { Lichenostomus cratitius } \\
\text { Purple-gaped }\end{array}$} \\
\hline Honeyeater & 2 & 2 & seeds & 1.29225607 & 4 & 0.418361 \\
\hline \multicolumn{7}{|l|}{ Lichenostomus penicillatus } \\
\hline Honeyeater & 2 & 2 & seeds & 1.29666519 & 4 & 0.462452 \\
\hline $\begin{array}{l}\text { Phylidonyris } \\
\text { novaehollandiae } \\
\text { New Holland }\end{array}$ & & & & & & \\
\hline Honeyeater & 2 & 2 & nectar & 1.30103 & 4 & 0.5061 \\
\hline \multicolumn{7}{|l|}{ Melanodryas cucullata } \\
\hline \multicolumn{7}{|l|}{ Chrysococcyx basalis } \\
\hline Horsfield's Bronze & & & & & & \\
\hline Cuckoo & 1 & 2 & seeds & 1.35793485 & 4 & 1.044632 \\
\hline \multicolumn{7}{|l|}{$\begin{array}{l}\text { Lichenostomus leucotis } \\
\text { White-eared }\end{array}$} \\
\hline Honeyeater & 2 & 2 & nectar & 1.36361 & 4 & 0.98786 \\
\hline \multicolumn{7}{|l|}{ Myiagra inquieta } \\
\hline $\begin{array}{l}\text { Restless Flycatcher } \\
\text { Lichenostomus virescens }\end{array}$ & 2 & 2 & inverts & 1.38021 & 4 & 0.82187 \\
\hline \multicolumn{7}{|l|}{ Lichenostomus virescens } \\
\hline Chrysocoecyx lucidus & & & & & & \\
\hline Shining Bronze Cuckoo & 2 & 2 & inverts & 1.39445 & 4 & 0.67946 \\
\hline
\end{tabular}


Table 1. (Continued)

\begin{tabular}{|c|c|c|c|c|c|c|}
\hline Species & $\begin{array}{l}\text { Nomad } \\
\text { Status } \\
\text { (Schodde) }\end{array}$ & $\begin{array}{l}\text { Nomad } \\
\text { Status (Reid) }\end{array}$ & Diet & $\begin{array}{l}\text { Log body } \\
\text { mass }\end{array}$ & Lump & Edge \\
\hline \multicolumn{7}{|l|}{ Circlorhamphus mathewsi } \\
\hline Rufous Songlark & 2 & 2 & seeds & 1.39794 & 4 & 0.64458 \\
\hline \multicolumn{7}{|l|}{ Certhionyx variegates } \\
\hline Pied Honeyeater & 1 & 1 & seeds & 1.41414 & 4 & 0.48261 \\
\hline \multicolumn{7}{|l|}{ Lalage sueurii } \\
\hline White-winged Triller & 2 & 2 & seeds & 1.41497 & 4 & 0.47425 \\
\hline \multicolumn{7}{|l|}{ Rhipidura leucophrys } \\
\hline Willie Wagtail & 2 & 2 & seeds & 1.44248 & 4 & 0.19918 \\
\hline \multicolumn{7}{|l|}{ Chrysococcyx osculans } \\
\hline Black-eared Cuckoo & 2 & 2 & inverts & 1.45818 & 4 & 0.04214 \\
\hline \multicolumn{7}{|l|}{ Merops ornatus } \\
\hline Rainbow Bee-eater & 2 & 2 & inverts & 1.45939 & 4 & 0.03006 \\
\hline \multicolumn{7}{|l|}{ Melopsittacus undulates } \\
\hline Budgerigar & 1 & 1 & seeds & 1.46240 & 4 & 0 \\
\hline \multicolumn{7}{|l|}{ Pachycephala inornata } \\
\hline Gilbert's Whistler & 2 & 2 & plant & 1.51455 & 5 & 0 \\
\hline \multicolumn{7}{|l|}{ Pachycephala pectoralis } \\
\hline Golden Whistler & 2 & 2 & seeds & 1.51455 & 5 & 0 \\
\hline \multicolumn{7}{|l|}{ Pomatostomus superciliosus } \\
\hline White-browed Babbler & 2 & 2 & seeds & 1.54407 & 5 & 0.24134 \\
\hline \multicolumn{7}{|l|}{ Artamus cinereus } \\
\hline Woodswallow & 1 & 2 & seeds & 1.54407 & 5 & 0.24134 \\
\hline \multicolumn{7}{|l|}{ Artamus personatus } \\
\hline Masked Woodswallow & 2 & 1 & nectar & 1.54937 & 5 & 0.18831 \\
\hline \multicolumn{7}{|l|}{ Geopelia cuneata } \\
\hline Diamond Dove & 1 & 1 & seeds & 1.55023 & 5 & 0.17973 \\
\hline \multicolumn{7}{|l|}{ Drymodes brunneopygia } \\
\hline Southern Scrub-robin & 2 & 2 & seeds & 1.56820 & 5 & 0 \\
\hline \multicolumn{7}{|l|}{ Climacteris picumnus } \\
\hline Brown Treecreeper & 2 & 2 & inverts & 1.56820 & 5 & 0 \\
\hline \multicolumn{7}{|l|}{ Artamus superciliosus } \\
\hline Woodswallow & 2 & 2 & seeds & 1.60206 & 6 & 0 \\
\hline \multicolumn{7}{|l|}{ Artamus cyanopterus } \\
\hline Dusky Woodswallow & 1 & 2 & seeds & 1.60206 & 6 & 0 \\
\hline \multicolumn{7}{|l|}{ Turnix velox } \\
\hline Little Button-quail & 1 & 1 & seeds & 1.61278 & 6 & 0.10724 \\
\hline \multicolumn{7}{|l|}{ Todiramphus sanctus } \\
\hline Sacred Kingfisher & 2 & 2 & inverts & 1.62014 & 6 & 0.18076 \\
\hline Neophema elegans & & & & & & \\
\hline Elegant Parrot & 1 & 2 & seeds & 1.63347 & 6 & 0.31409 \\
\hline $\begin{array}{l}\text { Glassopsitta } \\
\text { porphyrocephala }\end{array}$ & & & & & & \\
\hline Purple-crowned & & & & & & \\
\hline Lorikeet & 1 & 1 & nectar & 1.64147 & 6 & 0.39414 \\
\hline $\begin{array}{l}\text { Acanthagenys rufogularis } \\
\text { Sniny-ced }\end{array}$ & & & & & & \\
\hline Honeyeater & 1 & 2 & seeds & 1.64345 & 6 & 0.41393 \\
\hline Neophema chrysostoma & & & & & & \\
\hline Blue-winged Parrot & 1 & 2 & seeds & 1.65321 & 6 & 0.51153 \\
\hline Apus pacificus & & & & & & \\
\hline Fork-tailed Swift & 2 & 2 & inverts & 1.65610 & 6 & 0.54038 \\
\hline
\end{tabular}


Table 1. (Continued)

\begin{tabular}{|c|c|c|c|c|c|c|}
\hline Species & $\begin{array}{l}\text { Nomad } \\
\text { Status } \\
\text { (Schodde) }\end{array}$ & $\begin{array}{l}\text { Nomad } \\
\text { Status (Reid) }\end{array}$ & Diet & $\begin{array}{l}\text { Log body } \\
\text { mass }\end{array}$ & Lump & Edge \\
\hline \multicolumn{7}{|l|}{ Cacomantis flabelliformis } \\
\hline Fan-tailed Cuckoo & 2 & 2 & inverts & 1.67943 & 6 & 0.77368 \\
\hline \multicolumn{7}{|l|}{ Aegotheles cristatus } \\
\hline $\begin{array}{l}\text { Australian Owlet- } \\
\text { nightjar }\end{array}$ & 2 & 2 & inverts & 1.69897 & 6 & 0.96910 \\
\hline \multicolumn{7}{|l|}{ Todiramphus pyrrhopygia } \\
\hline Red-backed Kingfisher & 2 & 2 & inverts & 1.71858 & 6 & 1.16525 \\
\hline \multicolumn{7}{|l|}{ Geopelia striata } \\
\hline Peaceful Dove & 2 & 2 & inverts & 1.74818803 & 6 & 1.46128 \\
\hline \multicolumn{7}{|l|}{ Pomatostomus ruficeps } \\
\hline Babbler & 2 & 2 & seeds & 1.74818803 & 6 & 1.46128 \\
\hline \multicolumn{7}{|l|}{ Psephotus varius } \\
\hline \multirow{2}{*}{\multicolumn{7}{|c|}{ Oreoica gutturalis }} \\
\hline & & & & & & \\
\hline Crested Bellbird & 2 & 2 & 4 & 1.79239169 & 6 & 1.7749 \\
\hline \multicolumn{7}{|l|}{ Psephotus haematonotus } \\
\hline Red-rumped Parrot & 2 & 2 & seeds & 1.81291336 & 6 & 1.569683 \\
\hline \multicolumn{7}{|l|}{ Manorina flavigula } \\
\hline Yellow-throated Miner & 2 & 2 & seeds & 1.82930377 & 6 & 1.405779 \\
\hline \multicolumn{7}{|l|}{ Cinclosoma castanotus } \\
\hline Chestnut Quail-thrush & 2 & 2 & seeds & 1.86540012 & 6 & 1.044815 \\
\hline \multicolumn{7}{|l|}{ Colluricincla harmonica } \\
\hline Grey Shrike-thrush & 2 & 2 & 4 & 1.8785218 & 6 & 0.913598 \\
\hline \multicolumn{7}{|l|}{ Glossopsitta concinna } \\
\hline Musk Lorikeet & 1 & 1 & nectar & 1.87966921 & 6 & 0.902124 \\
\hline \multicolumn{7}{|l|}{ Northiella haematogaster } \\
\hline Blue Bonnet & 2 & 2 & nectar & 1.90308999 & 6 & 0.667917 \\
\hline \multicolumn{7}{|l|}{ Cuculus pallidus } \\
\hline Pallid Cuckoo & 2 & 2 & nectar & 1.9339426 & 6 & 0.35939 \\
\hline \multicolumn{7}{|l|}{ Turnix varia } \\
\hline Painted Button-quail & 2 & 2 & seeds & 1.94448267 & 6 & 0.25399 \\
\hline \multicolumn{7}{|l|}{ Eurostopodus argus } \\
\hline Spotted Nightjar & 2 & 2 & inverts & 1.94487731 & 6 & 0.250043 \\
\hline \multicolumn{7}{|l|}{ Nymphicus hollandicus } \\
\hline Cockatiel & 1 & 1 & seeds & 1.94748274 & 6 & 0.223989 \\
\hline \multicolumn{7}{|l|}{ Grallina cyanoleuca } \\
\hline Magpie-lark & 2 & 2 & seeds & 1.94939001 & 6 & 0.204916 \\
\hline \multicolumn{7}{|l|}{ Cracticus torquatus } \\
\hline Grey Butcherbird & 2 & 2 & seeds & 1.96567197 & 6 & 0.042097 \\
\hline \multicolumn{7}{|l|}{ Coracina novaehollandiae } \\
\hline $\begin{array}{l}\text { Black-faced Cuckoo- } \\
\text { shrike }\end{array}$ & 1 & 1 & seeds & 1.96988 & 6 & 0 \\
\hline Barnardius zonarius & & & & & & \\
\hline Australian Ringneck & 2 & 2 & seeds & 2.07188 & 7 & 0 \\
\hline $\begin{array}{l}\text { Hirundapus caudacutus } \\
\text { White-throated }\end{array}$ & & & & & & \\
\hline Needletail & 2 & 2 & inverts & 2.07918 & 7 & 0.07299 \\
\hline Trichoglossus haematodus & & & & & & \\
\hline Rainbow Lorikeet & 1 & 1 & nectar & 2.08636 & 7 & 0.14478 \\
\hline Anthochaera carunculata & & & & & & \\
\hline Red Wattlebird & 1 & 2 & nectar & 2.09691 & 7 & 0.25028 \\
\hline
\end{tabular}


Table 1. (Continued)

\begin{tabular}{|c|c|c|c|c|c|c|}
\hline Species & $\begin{array}{l}\text { Nomad } \\
\text { Status } \\
\text { (Schodde) }\end{array}$ & $\begin{array}{l}\text { Nomad } \\
\text { Status (Reid) }\end{array}$ & Diet & $\begin{array}{l}\text { Log body } \\
\text { mass }\end{array}$ & Lump & Edge \\
\hline \multicolumn{7}{|l|}{ Coracina maxima } \\
\hline Ground Cuckoo-shrike & 1 & 2 & inverts & 2.12574 & 7 & 0 \\
\hline \multicolumn{7}{|l|}{ Falco cenchroides } \\
\hline Nankeen Kestrel & 1 & 2 & inverts & 1.19312 & 8 & 0 \\
\hline \multicolumn{7}{|l|}{ Ninox novaeseelandiae } \\
\hline Southern Boobook & 2 & 2 & inverts & 2.24055 & 8 & 0.47425 \\
\hline \multicolumn{7}{|l|}{ Accipiter cirrhocephalus } \\
\hline Collared Sparrowhawk & 1 & 2 & inverts & 2.25527 & 8 & 0.45758 \\
\hline \multicolumn{7}{|l|}{ Ocyphaps lophotes } \\
\hline Crested Pigeon & 2 & 2 & seeds & 2.26435 & 8 & 0.36685 \\
\hline \multicolumn{7}{|l|}{ Phaps elegans } \\
\hline Brush Bronzewing & 2 & 2 & seeds & 2.30103 & 8 & 0 \\
\hline \multicolumn{7}{|l|}{ Elanus axillaris } \\
\hline Black-shouldered Kite & 1 & 1 & inverts & 2.39794 & 9 & 0 \\
\hline \multicolumn{7}{|l|}{ Falco longipennis } \\
\hline Australian Hobby & 1 & 2 & inverts & 2.40312 & 9 & 0.05181 \\
\hline \multicolumn{7}{|l|}{ Phaps chalcoptera } \\
\hline Common Bronzewing & 2 & 2 & seeds & 2.49136 & 9 & 0.93422 \\
\hline \multicolumn{7}{|l|}{ Gymmorhina tibicen } \\
\hline Australian Magpie & 2 & 2 & seeds & 2.49693 & 9 & 0.98990 \\
\hline \multicolumn{7}{|l|}{ Cacatua roseicappilla } \\
\hline Galah & 1 & 2 & seeds & 2.51248 & 9 & 1.14544 \\
\hline \multicolumn{7}{|l|}{ Podargus strigoides } \\
\hline Tawny Frogmouth & 2 & 2 & seeds & 2.54407 & 9 & 0.89400 \\
\hline \multicolumn{7}{|l|}{ Corcorax melanorhamphos } \\
\hline White-winged Chough & 2 & 2 & seeds & 2.56110 & 9 & 0.72367 \\
\hline \multicolumn{7}{|l|}{ Corvus bennetti } \\
\hline Little Crow & 1 & 1 & seeds & 2.58092 & 9 & 0.52544 \\
\hline \multicolumn{7}{|l|}{ Circus assimilis } \\
\hline Spotted Harrier & 1 & 1 & inverts & 2.62325 & 9 & 0.10219 \\
\hline \multicolumn{7}{|l|}{ Calyptorhynchus lathami } \\
\hline Glossy Black-Cackatoo & 1 & 2 & seeds & 2.63347 & 9 & 0 \\
\hline \multicolumn{7}{|l|}{ Accipiter fasciatus } \\
\hline Brown Goshawk & 1 & 2 & inverts & 2.70757 & 10 & 0 \\
\hline \multicolumn{7}{|l|}{ Tyto alba } \\
\hline Barn Owl & 1 & 1 & inverts & 2.71892 & 10 & 0.11347 \\
\hline \multicolumn{7}{|l|}{ Cacatua sanguinea } \\
\hline Little Corella & 1 & 2 & seeds & 2.72016 & 10 & 0.12589 \\
\hline Falco berigora & & & & & & \\
\hline Brown Falcon & 1 & 1 & inverts & 2.73997 & 10 & 0.32398 \\
\hline Corvus mellori & & & & & & \\
\hline Little Raven & 1 & 2 & inverts & 2.75358 & 10 & 0.46013 \\
\hline Tyto novaehollandiae & & & & & & \\
\hline Masked Own & 2 & 2 & inverts & 2.78462 & 10 & 0.77047 \\
\hline Carvus coronoides & & & & & & \\
\hline Australian Raven & 2 & 2 & seeds & 2.82930 & 10 & 0.94458 \\
\hline Burhinus grallarius & & & & & & \\
\hline Bush Stone Curlew & 1 & 2 & plant & 2.83554 & 10 & 0.88217 \\
\hline Falco subniger & & & & & & \\
\hline Black Falcon & 1 & 1 & inverts & 2.89515 & 10 & 0.28616 \\
\hline Haliastur sphenurus & & & & & & \\
\hline Whistling Kite & 1 & 2 & inverts & 2.90309 & 10 & 0.20672 \\
\hline
\end{tabular}


Table 1. (Continued)

\begin{tabular}{|c|c|c|c|c|c|c|}
\hline Species & $\begin{array}{l}\text { Nomad } \\
\text { Status } \\
\text { (Schodde) }\end{array}$ & $\begin{array}{l}\text { Nomad } \\
\text { Status (Reid) }\end{array}$ & Diet & $\begin{array}{l}\text { Log body } \\
\text { mass }\end{array}$ & Lump & Edge \\
\hline \multicolumn{7}{|l|}{ Calyptorhynchus funereus } \\
\hline \multicolumn{7}{|l|}{ Yellow-tailed Black } \\
\hline Cockatoo & 1 & 2 & seeds & 2.90363 & 10 & 0.20129 \\
\hline \multicolumn{7}{|l|}{ Milvus migrans } \\
\hline Black Kite & 1 & 1 & inverts & 2.91751 & 10 & 0.06257 \\
\hline \multicolumn{7}{|l|}{ Hieraaetus morphnoides } \\
\hline Little Eagle & 1 & 2 & inverts & 2.92376 & 10 & 0 \\
\hline \multicolumn{7}{|l|}{ Leipoa ocellata } \\
\hline Malleefowl & 2 & 2 & plant & 3.27254 & 11 & 0 \\
\hline \multicolumn{7}{|l|}{ Aquila audax } \\
\hline Wedge-tailed Eagle & 1 & 2 & inverts & 3.54407 & 11 & 2.71530 \\
\hline \multicolumn{7}{|l|}{ Dromaius novaehollandiae } \\
\hline Emu & 1 & 2 & plant & 4.49360 & 11 & 0 \\
\hline $\begin{array}{l}\text { Nomenclature follows Christidis a } \\
\text { Two different interpretations of no } \\
\text { "Diet" is split into four categories } \\
\text { "Lump" refers to the identified bo } \\
\text { "Edge" refers to the distance, in } b\end{array}$ & $\begin{array}{l}\text { (1994). } \\
\text { le been used, }-S c \mid \\
\text { nvertebrates (inve } \\
\text { aggregations. } \\
\text { terms, of a specic }\end{array}$ & $\begin{array}{l}\text { 81) and Saunders and } \\
\text { rr, and plants. } \\
\text { body mass aggregation }\end{array}$ & id (Reid); in & a 1 is used for & & \\
\hline
\end{tabular}

The body mass distribution of the bird community studied was discontinuous. We identified 11 significant aggregations of species. The body mass structure of those communities is given in Table 1 .

Because of the small number of species that were categorized as plant feeders, the variable plant was removed from our models. Using the nomad classification scheme of Schodde, the backward selection process selected a significant $(P=0.0001)$ twovariable model (Table 2 ). The significant variables were body mass $(P=0.001)$ and proximity to body mass aggregation edge $(P=0.011)$. The variable nectar $(P=0.11)$ approached significance. Observed concordance in responses was $73 \%$. The Hosmer and Lemeshow goodness-of-fit test statistic $(9.3,8 \mathrm{df}, P=0.32)$ indicates that the null hypothesis that the model fits the data well cannot be rejected. We also tested for model fit by comparing AIC values (Akaike 1969) for all possible models (all variable and interaction combinations) and confirmed that the two variable model was the best fit. The final backward selection model took the following form:

$$
\begin{aligned}
& \text { Logit }(\text { nomad })=-2.317 \cdot \text { intercept } \\
& +1.313 \cdot \text { mass }-1.207 \cdot \text { edge distance }
\end{aligned}
$$

Using the nomad classification scheme of Saunders and Reid, the backward selection process also converged upon a significant $(P=0.03)$ two-variable model (Table 3 ). The significant variables were nectar diet $(P=0.075)$ and proximity to body mass aggregation edge $(P=0.086)$. Observed concordance in response was $62 \%$. The Hosmer and Lemeshow goodness-of-fit test statistic $(5.6,8 \mathrm{df}$, $P=0.69$ ) indicates that the null hypothesis that the model fits the data well cannot be rejected. The final backward selection model took the following form:

$$
\begin{aligned}
& \text { Logit }(\text { nomad })=0.893 \cdot \text { intercept } \\
& \quad-1.043 \cdot \text { pollen }-1.184 \cdot \text { edge distance }
\end{aligned}
$$

\section{DisCUSSION}

Nomadism as an ecological phenomenon in birds, humans, and other animals has vaguely been explained away as a response to variable environments. Although this is undoubtedly true, it does little to address the basic question; Given a suite of species in a variable environment, what predicts which species will be nomadic?

Nomadic species in the Lofty Block of southcentral Australia were associated with the edge of body mass aggregations using two divergent and independent classification schemes. The Schodde classification scheme also yielded mass as a significant 
Table 2. Backward Selection Logistic Regression Model of Predictors of Nomadism in the Mallee of Southcentral Australia, Based on the Classification of Schodde (1981)

\begin{tabular}{llllll}
\hline Variable & $\begin{array}{l}\text { Parameter } \\
\text { Estimate }\end{array}$ & $\begin{array}{l}\text { Standard } \\
\text { Error }\end{array}$ & $\begin{array}{l}\text { Wald } \\
\text { Chi-square }\end{array}$ & $P$ Value & $\begin{array}{l}\text { Odds } \\
\text { Ratio }\end{array}$ \\
\hline Intercept & -2.3169 & 0.6185 & 14.0341 & 0.0002 & na \\
Mass & 1.3133 & 0.3394 & 14.9691 & 0.0001 & 3.718 \\
Edge Distance & -1.2068 & 0.4761 & 6.4242 & 0.0113 & 0.299 \\
\hline
\end{tabular}

Table 3. Backward Selection Logistic Regression Model of Predictors of Nomadism in the Mallee of Southcentral Australia, Based on the Classification of Saunders and Reid

\begin{tabular}{llllll}
\hline Variable & $\begin{array}{l}\text { Parameter } \\
\text { Estimate }\end{array}$ & $\begin{array}{l}\text { Standard } \\
\text { Error }\end{array}$ & $\begin{array}{l}\text { Wald } \\
\text { Chi-square }\end{array}$ & $P$ Value & $\begin{array}{l}\text { Odds } \\
\text { Ratio }\end{array}$ \\
\hline Intercept & 0.8929 & 1.0971 & 0.6624 & 0.4157 & na \\
Nectar & -1.0429 & 0.5848 & 3.1806 & 0.0745 & 0.352 \\
Edge Distance & -1.1844 & 0.6908 & 2.9397 & 0.0864 & 0.306 \\
\hline
\end{tabular}

predictor of nomadism. In that model, the closer a species is, in body mass terms, to a body mass aggregation edge, and the larger a species is, the more likely it is to be nomadic. Using the classification scheme of Saunders and Reid, body mass was not a predictor of nomadism but a nectar diet was. The Schodde categorization scheme lent itself better to logistic model building, simply because the response variable was more approximately evenly distributed between the response (nomadic) and no-response states; the classification scheme of Saunders and Reid considered $50 \%$ fewer species to be nomadic. However, the association of nomadic species with body mass aggregation edges was robust despite these large differences in the categorization of nomadism.

Allen and others (1999) documented that invasions and extinctions in the vertebrate fauna of south Florida and Australia were associated with the edge of body mass aggregations. Invasion and extinction represents community-level turnover in species composition, while nomadism represents population-level, temporal turnover. All these phenomena reflect high variability in animal communities at the ecosystem level and are distributed nonrandomly in terms of body mass distributions. Allen and others (1999) hypothesized that the edge of body mass aggregations were scale breaks and analogous to phase transitions between one range of scale of landscape pattern and another. They predicted that other biological phenomena indicative of high variability would be associated with the edge of body mass aggregations. The occurrence of nomadic species at scale breaks confirms that pre- diction and suggests greater variability in resources at scale breaks.

We suggest that this reflects a response to ecological patterns that vary with scale. At larger scales that pattern may be largely due to the spatial configuration of patches (Morton 1990), but at small scales that pattern is due to the architecture of twigs, branches, and other vegetation. An animal cannot simultaneously interact with multiple scales of pattern; it must either specialize at a single scale or shift back and forth between two scales. The scales of pattern available in an ecosystem, determined by the scale domains of key processes interacting (self-organizing) with existing structure, determine the number of body mass aggregations in an animal body mass distribution. The number of species within a given body mass aggregation should reflect the overall availability of resources at that scale. The change from one scale of pattern to another is not continuous; rather, it is abrupt, without transition. If this is the case, having a body mass that places an animal in between scales will not be adaptive, since there is no resource pattern or ecological structure with which to interact, and this absence of pattern will cause the gaps observed in body mass patterns, either through species assortment or character (mass) displacement. However, exploiting the environment close to these scale breaks, and perhaps being able to flip back and forth between scales, may be advantageous, especially in highly variable systems.

The evidence for a link between landscape structure at different scales and body mass patterns is critical for this argument. However, because the 
manipulation of entire ecosystems at multiple scales with replication is a challenge, an approach that employs the process of adaptive inference (Holling and Allen 2002) is needed to establish the most likely causative mechanisms for lumpiness and to determine whether or not a link exists between body mass aggregations and landscape structure. Discontinuous body mass patterns have been documented in all systems investigated (see, for example, Holling 1992; Restrepo and others 1997; Lambert and Holling 1998; Allen and others 1999; Raffaelli and others 2000; Havlicek and Carpenter 2001). The occurrence of discontinuities and aggregations in body mass distributions appears to be the rule. The analyses of Raffaelli and others (2000), Havlicek and Carpenter (2001), and Forys and Allen (2002) suggest that body mass structure is a conservative property, robust to both perturbations and large turnover in community composition. The association of attributes of species independent of the determination of body mass structure, invasiveness and decline (Allen and others 1999), and nomadism provide further strong evidence that the pattern identified in body mass distributions is not a statistical artifact. The analysis of landscape patterns has demonstrated that changes in spatial pattern across different ranges of scales are described by different scaling relationships (Krummel and others 1987). Furthermore, the analysis of the relationship between species richness and spatial scale has shown the existence of different scaling relationships at different scales (Crawley and Harral 2001). In between scales, there are sharp breaks between these scaling regimes (Krummel and others 1987; Crawley and Harral 2001). A direct link between body mass aggregations and landscape pattern remains elusive, but others have demonstrated a relationship between body mass and vegetation structure (Morse and others 1988; Shorrocks and others 1991). Also, Sendzimir (unpublished) has established a qualitative relationship between body mass aggregations and the scale and texture of mammal landscape use. Raffaelli and others (2000) conducted manipulations of marine sediment invertebrate assemblages and demonstrated that while perturbations affected densities and abundance of taxa, aggregations and gaps in size spectra were conserved. They concluded that the size spectrum was probably constrained by habitat structure-a finding that strongly supports the textural discontinuity hypothesis of Holling (1992). Thus, the weight of current evidence supports the proposition that body mass patterns are entrained by, and correspond to, landscape structure at different scales.

The evolution of nomadism may be an adaptive response to increased variability following environmental change experienced by species living at affected scales, or it may be an adaptive response of species forced to less optimal scales and habitats by interspecific interactions. In systems with high inherent resource variability, species that exploit resources between distinct ranges of scales may be especially prone to the evolution of nomadic behavior. As resources bottleneck and general variability increase, species strategies may become more inflexible. Species must either specialize within a single scale-if body size, competitive interactions, and resource distributions allow it-or they will be forced into nomadism. In humans, nomadism occurs at the periphery of settled heartlands (Rosen 1988), but this "marginal" existence may lead to innovation, such as the development of animal domestication. A combination of variability from extrinsic environmental factors and the intrinsic variability inherent in exploiting the environment at or near scale breaks may be especially fertile for the evolution of complex behaviors. The existence of general, flexible behaviors, such as those represented by nomadism, across multiple scales may add to the resilience of ecosystems facing rapid environmental change.

\section{ACKNOWLEDGMENTS}

We thank J. Reid for providing expertise regarding the nomadic status of the birds of the Lofty Block, R. Schodde for providing raw weights for a number of species for which published data were not available, and T. Jenner for preparing the figure inset. This paper was improved by incorporating comments from E. Forys and two anonymous reviewers. The South Carolina Cooperative Fish and Wildlife Research Unit is jointly supported by a cooperative agreement among the USGS/BRD, the South Carolina Department of Natural Resources, Clemson University, and the Wildlife Management Institute.

\section{REFERENCES}

Akaike H. 1969. Fitting autoregressive models for prediction. Ann Inst Stat Math 21:243-7.

Allen CR, Forys EA, Holling CS. 1999. Body mass patterns predict invasions and extinctions in transforming landscapes. Ecosystems 2:114-21.

Christidis L, Boles WE. 1994. The taxonomy and species of birds of Australia and its territories. Royal Australasian Ornithologists Union monograph 2. Melbourne: Royal Australasian Ornithologists Union.

Crawley MJ, Harral JE. 2001. Scale dependence in plant biodiversity. Science 291:864-8.

Davies SJJF. 1984. Nomadism as a response to desert conditions in Australia. J Arid Environ 7:183-95. 
Dean WRJ. 1997. The distribution and biology of nomadic birds in the Karoo, South Africa. J Biogeog 24:769-79.

Dunning JB Jr. 1993. CRC handbook of avian body masses. Ann Arbor (MI): CRC Press.

Eisenberg JF. 1981. The mammalian radiations: an analysis of trends in evolution, adaptation and behavior. Chicago: University of Chicago Press.

Forys EA, Allen CR. 2002. Functional group change within and across scales following invasions and extinctions in the Everglades ecosystem. Ecosystems. 5:339-47.

Havlicek T, Carpenter SR. 2001. Pelagic size distributions in lakes: are they discontinuous? Limnol Oceanogr. 46:10211033.

Holling CS. 1992. Cross-scale morphology, geometry, and dynamics of ecosystems. Ecol Monog 62:447-502.

Holling CS, Allen CR. 2002. Adaptive inference for distinguishing credible from incredible patterns in nature. Ecosystems 5:319-328.

Keast A. 1968. Seasonal movements in the Australian honeyeaters (Meliphagidae) and their ecological significance. Emu 67:159-209.

Krummel JR, Gardner RH, Sugihara G, O'Neill RV, Coleman PR. 1987. Landscape patterns in a disturbed environment. Oikos 48:321-4

Lambert WD, Holling CS. 1998. Causes of ecosystem transformation at the end of the Pleistocene: evidence from mammal body mass distributions. Ecosystems 1:157-75.

Lasiewski RC, Dawson WR. 1967. A re-examination of the relation between standard metabolic rate and body weight in birds. Condor 69:13-23.

Maclean GL. 1996. Ecophysiology of desert birds. New York: Springer-Verlag.

Menard S. 1995. Applied logistic regression analysis. Sage University Series on Quantitative Applications in the Social Sciences, 07-106. Thousand Oaks (CA): Sage Press.

Morse DR, Stork NE, Lawton JH. 1988. Species number, species abundance and body length relationship of arboreal beetles in Bornean lowland rainforest trees. Ecol Entomol 13:25-37.

Morton SR. 1990. The impact of European settlement on the vertebrate animals of arid Australia: a conceptual model. Proc Ecol Soc Aust 16:201-13.
Paton DC, Prescott AM, Davies RJ-P, Heard LM. 2000. The distribution, status and threats to temperate woodlands in South Australia. In: Hobbs RJ, Yates CJ, editors. Temperate eucalypt woodlands in Australia. Chipping Norton (Australia): Surrey Beatty \& Sons. p 57-85.

Peterson GD, Allen CR, Holling CS. 1998. Ecological resilience, biodiversity and scale. Ecosystems 1:6-18.

Raffaelli D, Hall S, Emes C, Manly B. 2000. Constraints on body size distributions: an experimental approach using a smallscale system. Oecologia 122:389-98.

Restrepo C, Renjifo LM, Marples P. 1997. Frugivorous birds in fragmented neotropical montane forests: landscape pattern and body mass distribution. In: Laurance WF, Bierregaard RO, Moritz C, editors. Tropical forest remnants: ecology, management and conservation of fragmented communities. Chicago: University of Chicago Press. p 171-89.

Rosen SA. 1988. Notes on the origins of pastoral nomadism: a case study from the Negev and Sinai. Curr Anthropol 29:498506.

SAS Institute. Inc. 1989. SAS/STAT user's guide. Version 6, 4th ed. Cary (NC): SAS Institute.

Schodde R. 1981. Bird communities of the Australian mallee: composition, derivation, distribution, structure and seasonal cycles. In: di Castri F, Goodall DW, Specht RL, editors. Mediterranean-type shrublands. New York: Elsevier Scientific. $p$ 387-415.

Shorrocks B, Marsters J, Ward I, Evernett PJ. 1991. The fractal dimension of lichens and the distribution of arthropod body lengths. Funct Ecol 5:457-60.

Silverman BW. 1981. Using kernel density estimates to investigate multimodality. J R Stat Soc 43:97-9.

Sinclair ARE. 1984. The function of distance movements in vertebrates. In: Swingland IR, Greenwood PJ, editors. The ecology of animal movement. Oxford (England): Clarendon Press. p 240-58.

Thackway R, Cresswell ID, editors. 1995. An interim biogeographic regionalisation for Australia. Canberra: Australian Nature Conservation Agency.

Wiens JA. 1991. Ecological similarity of shrub-desert avifaunas of Australia and North America. Ecology 72:479-95. 